\title{
PRIMING SINTÁCTICO EN LA PRODUCCIÓN DE ORACIONES EN ESPAÑOL. ACERCA DE LA REPRESENTACIÓN SINTÁCTICA DE LOS VERBOS
}

SYNTACTIC PRIMING IN SENTENCE PRODUCTION IN SPANISH. ABOUT THE SYNTACTIC REPRESENTATION OF VERBS

\author{
YAMILA SEVILLA*** \\ yamilasevilla@gmail.com \\ VIRGINIA JAICHENCO ${ }^{* *}$ \\ vjaichen@psi.uba.ar \\ MAXIMILIANO WILSON ${ }^{* * *}$ \\ maximiliano.wilson@istc.cnr.it \\ ALEJANDRO RAITER ${ }^{* *}$ \\ araiter@filo.uba.ar
}

\section{RESUMEN}

Este trabajo presenta una experiencia de priming sintáctico dirigida a investigar la información sintáctica representada en las entradas léxicas de los verbos. Mediante una tarea de completamiento escrito de oraciones que manipuló las propiedades combinatorias de un conjunto de verbos (que seleccionan o bien un Sintagma Nominal o bien un Sintagma de Flexión o bien un Sintagma de Complementante) se mostró que los participantes tendían a utilizar las estructuras que habían experimentado previamente al completar nuevas oraciones. Este efecto se verificó con la misma intensidad cuando los facilitadores eran oraciones que los participantes habían producido (Condición II), como cuando se trataba de oraciones que habían leído (Condición I), lo que confirma hallazgos previos (i.e. Bock, 2002; Bock et al., 2007) en cuanto a que el priming tiene lugar de comprensión a producción. El experimento también mostró que el priming era sensible a la distinción SC/SF pero no a la estructura interna de esos constituyentes. Este hallazgo permite inferir que la información combinatoria (Pickering y Branigan, 1998) asociada a los nodos lema de los verbos (Levelt, Roelofs y Meyer, 1999) es de nivel sintagmático y que codifica la naturaleza categorial de los sintagmas que el verbo selecciona.

Palabras clave: Psicolingüística, priming sintáctico, producción de oraciones, verbos, propiedades combinatorias.

\footnotetext{
*Consejo Nacional de Investigaciones Científicas y Técnicas.

${ }^{* *}$ Universidad de Buenos Aires. Argentina.

${ }^{* * *}$ Institute for Cognitive Sciences and Technologies (ISTC), CNR, Roma, Italia.
} 


\section{ABSTRACT}

We present the results of a syntactic priming experiment aimed at investigating the syntactic information represented in the lexical entries of verbs. By means of a written sentence completion task that manipulated the combinatory properties of a set of verbs (by selecting either a NP or a IP or a CP) we found that participants tended to use the previously experienced structures in the new sentences. This effect was found either when the prime sentences were the ones participants had produced before (Condition II) or when primes were previously read sentences (Condition I). This confirms previous findings (i.e. Bock, 2002; Bock et al. 2007) in the sense that priming takes place from comprehension to production as well. These experiments also showed that priming was sensitive to CP/IP distinction but not to constituents' internal structure. These findings enable us to infer that combinatory information (Pickering y Branigan, 1998) associated with lemma nodes of the verbs (Levelt, Roelofs y Meyer, 1999) is at phrasal level, and that it codifies the categorial nature of the XP selected by the verb.

Keywords: Psycholinguistics, syntactic priming, sentence production, verbs, combinatory properties.

Recibido: 16-10-2007. Aceptado: 13-11-2008

\section{INTRODUCCIÓN}

$\mathrm{N}$ umerosos factores guían a los hablantes en la selección de una estructura sintáctica para expresar sus ideas mediante oraciones. Entre ellos, los factores discursivos y pragmáticos parecen tener una importancia crucial en el habla corriente. Sin embargo, los estudios sobre el procesamiento lingüístico han destacado el hecho de que los hablantes tienden a reutilizar estructuras que han experimentado previamente. Este patrón de conducta, conocido como 'priming sintáctico', había sido identificado en trabajos observacionales sobre corpora (Kempen, 1977; Shenkein, 1980; Weiner y Labov, 1983; Tannen, 1989) y fue más tarde estudiado sistemáticamente con enfoques experimentales.

Bock (1986) introdujo el paradigma experimental que luego otras investigaciones desarrollaron. En su trabajo pionero, los hablantes debían describir dibujos blancos que eran precedidos por oraciones facilitadoras no relacionadas semánticamente. La autora demostró que los participantes a menudo utilizaban de nuevo las estructuras que les habían sido presentadas en las oraciones facilitadoras. A este estudio le sucedieron muchos otros, que confirmaron el fenómeno utilizando distintas tareas, diversas estructuras sintácticas, diferentes lenguas, modalidades y paradigmas de experimentación (Bock, Loebell y Morey, 1992; Branigan, Pickering y Cleland, 2000; Hartsuiker y Kolk, 1998; Hartsuiker, Kolk y Huiskamp, 1999; Hartsuiker y Westenberg, 2000; Pickering y Branigan, 1998; Potter y Lombardi, 1998). Pickering y Branigan (1999) ofrecen una completa revisión de estos trabajos. 
En conjunto, los estudios muestran que, si bien es posible que factores no estructurales contribuyan parcialmente de acuerdo con el tipo de tarea y las características de los estímulos, el priming sintáctico no puede ser explicado por correspondencias métricas, semánticas, léxicas u otras similaridades superficiales entre facilitador y blanco. Por ejemplo, se mostró que oraciones superficialmente similares con estructuras de constituyentes diferentes no inducen efectos de priming (complementos oracionales con "to" no facilitan oraciones dativas con "to"; Bock y Loebell, 1990). Sin embargo, se observan efectos de priming cuando las oraciones prime y blanco poseen la misma estructura aunque no comparten palabras funcionales (sintagmas preposicionales con "for" facilitan sintagmas preposicionales con "to"; Bock, 1989).

El fenómeno fue utilizado para investigar distintos aspectos del procesamiento gramatical ${ }^{1}$. En el paradigma más usual, la facilitación se logra mediante la producción, por parte de los hablantes, de una oración con una estructura particular, típicamente obtenida a través de la lectura en voz alta o la repetición. Sin embargo, Potter y Lombardi (1998) mostraron mediante experimentos de recuerdo que el priming para la producción puede ocurrir como resultado de la comprensión sola, es decir sin que sea necesario producir previamente la estructura facilitadora. Los participantes de su estudio mostraron mayor probabilidad de recordar una estructura alternativa para una oración (por ejemplo la forma con preposición en vez de la forma de doble objeto en oraciones dativas) después de leer una oración con la estructura alternativa. Bock (2002) y Bock et al. (2007) aportaron nueva evidencia de que el priming estructural opera de comprensión a producción.

También Branigan et al. (2000) encontraron, en una experiencia que utilizaba el diálogo, que los participantes tendían a producir descripciones de imágenes con la misma estructura (objeto preposicional o doble objeto en oraciones dativas) que simplemente habían escuchado antes, utilizada por un cómplice encubierto del experimentador para dirigirse a ellos, o incluso a un tercer participante.

Como señala Branigan (2007), el priming sintáctico parece particularmente fuerte en el diálogo, razón por la que se ha propuesto que se trata de un mecanismo fundamental subyacente a la convergencia de los hablantes sobre representaciones semánticas para hacer exitosa la comunicación (Pickering y Garrod, 2004). Hay bastante evidencia de estudios observacionales y experimentales, en los distintos niveles, de que los participantes del diálogo tienden a coordinar conceptual y formalmente sus contribuciones. En este sentido, el priming sintáctico podría ser un medio para reducir la carga computacional asociada con el procesamiento sintáctico.

${ }^{1}$ Como el orden lineal de constituyentes (Hartsuiker, Kolk y Huiskamp, 1999; Hartsuiker y Westenberg, 2000) y las preferencias de adjunción de cláusulas relativas (Scheepers, 2003). Se ha mostrado, también, que el priming actúa tanto sobre la forma como sobre el tiempo de reacción (Corley y Scheepers, 2002; Smith y Wheeldon, 2001). 
Si bien su carácter estructural es ampliamente aceptado en la bibliografía, no hay aún acuerdo respecto de cuáles serían los mecanismos y niveles de representación responsables del priming sintáctico. Dos líneas de investigación han sido propuestas para explicar el fenómeno. Esquemáticamente, las explicaciones descansan o bien en la preservación de la configuración sintáctica de la oración facilitadora (Bock y Griffin, 2000) o bien en la recuperación de propiedades sintácticas asociadas con elementos léxicos en la oración inductora (Branigan, Pickering, Liversedge, Stewart y Urbach, 1995).

La primera de estas explicaciones concibe el priming como un mecanismo de largo plazo que ocasiona adaptaciones duraderas en el sistema de procesamiento del lenguaje. Bock y Griffin (2000) sugirieron que refleja un proceso de aprendizaje implícito, por el cual el patrón de asociación entre rasgos del mensaje a expresar y una estructura se refuerza durante el procesamiento. De este modo, la configuración sintáctica superficial dada y su procedimiento de construcción se vuelven más accesibles y tienden a ser reutilizados (i.e. Bock y Griffin, 2000; Bock y Loebell, 1990). Procesar un prime, así, causa ajustes en los pesos de las conexiones entre elementos de significado y estructura sintáctica, dando como resultado una tendencia a proyectar los mismos elementos de mensaje en las mismas configuraciones sintácticas (Chang, Dell, Bock y Griffin, 2000).

Esta perspectiva explica de manera natural la persistencia y la naturaleza aparentemente acumulativa del priming, así como la tendencia de las estructuras menos preferidas a primar más fuertemente que las estructuras preferidas (por ejemplo, Bock, 1986; Hartsuiker et al., 1999; Ferreira, 2003).

Sin embargo, hay evidencias que este modelo tiene dificultades para explicar. En particular, ¿por qué el priming surge de la exposición previa a una palabra aislada? Algunos estudios han mostrado que procesar un verbo aislado asociado con un marco de subcategorización particular es suficiente para primar la producción de una oración con esa estructura (Melinger y Dobel, 2005; Sevilla, Jaichenco, Olmedo y Raiter, 2006; ver además Trueswell y Kim, 1998). Dado que el modelo atribuye el efecto a la proyección de elementos del mensaje sobre estructuras sintácticas, no puede explicar cómo la exposición a una palabra aislada puede inducir priming para la estructura de una oración.

A partir de evidencias de este orden, diversos autores (Trueswell y Kim, 1998; Melinger y Dobel, 2005; entre otros) apoyan el rol mediador de los elementos léxicos. De acuerdo con Branigan et al. (1995) y Pickering y Branigan (1998), el efecto se debe a la activación residual de rasgos morfosintácticos léxicamente especificados, como por ejemplo los marcos de subcategorización asociados con los verbos en las oraciones facilitadoras.

Desde esta perspectiva, el priming sintáctico aporta evidencia acerca de la organización de la información sintáctica de las palabras. A partir de una serie de experimentos en los que utilizaban la alternancia dativa y variaban las condiciones 
en las que se inducía el priming, Pickering y Branigan (1998) desarrollaron una extensión del modelo del estrato del lema (Roelofs, 1992, 1993; Levelt, Roelofs y Meyer, 1999) para incluir, junto a otros nodos que codifican información sintáctica, como categoría o rasgos morfológicos (phi), un nodo que indica con qué elementos se puede combinar esa palabra. Ellos sugirieron que los nodos lema que representan verbos que pueden ser combinados con una forma sintáctica particular están directamente vinculados al mismo nodo combinatorio. De acuerdo con su modelo, la producción o comprensión de una expresión implica la activación del lema correspondiente en asociación con el nodo combinatorio apropiado; si el mismo nodo es relevante en el procesamiento subsiguiente, entonces la activación residual da lugar al priming sintáctico.

Como parte de una investigación que enfoca la información sintáctica representada en las entradas léxicas de los verbos, en este estudio nos propusimos analizar la naturaleza de la información combinatoria. Para ello utilizamos una experiencia de priming sintáctico basada en el completamiento escrito de oraciones con verbos (del tipo de "recomendar") que seleccionan sintácticamente o bien un sintagma de flexión (SF; como en "les recomendó solucionar el conflicto pacíficamente") o bien un sintagma de complementante (SC; como en "les recomendó que solucionaran el conflicto pacíficamente") o bien un sintagma nominal (SN; "les recomendó una solución pacífica del conflicto"). Nuestro experimento primó alternativamente las formas con SF y con SC que, aunque categorialmente diferentes, expresan eventos ${ }^{2}$. Si bien la elección de una u otra forma en una emisión natural puede verse afectada por otros factores como el contexto o las características sintácticas y semánticas de la cláusula subordinada, ${ }^{3}$ la alternancia de estas formas permite estudiar la organización de las propiedades de selección de los verbos.

Nuestro experimento se propuso investigar qué tipo de unidades codifican la información acerca de cómo combinar unas palabras con otras. La influencia de las tendencias de subcategorización del verbo ya fueron estudiadas previamente en comprensión de oraciones (Trueswell y Kim, 1998) y para la producción de oraciones con alternancia dativa en alemán y holandés (Melinger y Dobel, 2005). El presente estudio investiga el fenómeno en la producción de oraciones en español.

Por otra parte, mediante condiciones sucesivas, nuestro experimento buscó también establecer si hay diferencias en la magnitud del priming cuando este sólo involucra producción y cuando se produce de comprensión a producción.

\footnotetext{
${ }^{2}$ Nótese que, salvo una lista muy reducida de predicados que normalmente excluyen una completiva con verbo finito, la distribución de este tipo de estructuras como complemento de $\mathrm{V}$ es muy similar (Herranz, 1999).

${ }^{3}$ En particular, por la referencia del sujeto de la subordinada.
} 


\section{MÉTODO}

\subsection{Tarea:}

La tarea que los sujetos debían realizar consistió en el completamiento escrito de oraciones a partir de un fragmento que incluía un verbo crítico (ver apartados 2.3 y 2.4).

\subsection{Sujetos:}

El Experimento se administró a 32 sujetos adultos, de ambos sexos, entre 20 y 34 años de edad (media=24,7), universitarios, con un mínimo de 13 años de escolaridad. Además, 50 sujetos adultos de ambos sexos, con edades entre los 20 y 35 años (media=25,1), también universitarios, constituyeron el grupo control.

\subsection{Estímulos:}

Se utilizó un conjunto de verbos que permiten tres combinaciones sintácticas diferentes, de acuerdo con la naturaleza categorial del complemento: sintagma de flexión (SF), sintagma de complementante (SC) o sintagma nominal (SN). Así, los blancos para completar (fragmentos de oraciones) fueron construidos sobre un conjunto de verbos (como "recomendar") que seleccionan o bien un sintagma nominal (como en "recomendó una solución pacífica del problema”), o bien un sintagma de flexión (por ejemplo, "recomendó solucionar el problema pacíficamente") o bien un sintagma de complementante (como en "recomendó que solucionaran el problema pacíficamente").

Se confeccionaron 10 conjuntos de ítemes críticos, cada uno comprendía la siguiente secuencia: 1) una oración contextualizadora y una pregunta (facilitador 1) que los participantes debían leer; 2) un fragmento de oración (blanco 1, facilitador 2) que debía ser completado como respuesta a la pregunta; 3) un segundo fragmento de una oración no relacionada, con el mismo verbo (blanco 2), que debían también completar. Por ejemplo:

1. a) La DEA se entrevistó con los funcionarios colombianos.

¿Qué les recomendó que hicieran?

1. b) La DEA se entrevistó con los funcionarios colombianos.

¿Qué les recomendó hacer? 
2. La DEA les recomendó a los funcionarios colombianos...

\#

3. Durante la misa, el cura les recomendó a los fieles...

Se previeron 2 condiciones. En la Condición I -de comprensión a producción-, la pregunta ( $1 \mathrm{a}$ o $1 \mathrm{~b}$ ) funciona como inductora respecto de 2 , que es el fragmento blanco (blanco 1). La oración completada en 2 (facilitador 2) es, a su vez, el inductor respecto de 3 (blanco 2), en lo que constituye la Condición II -de producción a producción ${ }^{4}$.

Cada fragmento blanco contiene un sujeto, un complemento indirecto plural y el clítico duplicado correspondiente. Cada blanco del tipo 1 fue precedido de una pregunta de tipo a) en el $50 \%$ de los casos y de una pregunta de tipo b) en el $50 \%$ restante. La forma con SN no fue inducida.

Facilitador y blanco incluyen el mismo verbo con idéntica forma del verbo en las dos condiciones: tercera persona del singular, pretérito perfecto simple.

Los estímulos fueron presentados en un cuadernillo que contenía, entre cada secuencia relevante, dos secuencias de fragmentos de relleno (oraciones con estructuras variadas sin interés para la prueba). Estas secuencias de relleno debían completarse y tenían por finalidad impedir la persistencia de la facilitación en los fragmentos relevantes de una secuencia a otra. De esta manera, cada cuadernillo contaba con 20 fragmentos experimentales y 40 fragmentos de relleno.

\subsection{Procedimiento:}

Cada sujeto recibió un cuadernillo autoadministrable. La consigna no previó límite de tiempo, pero se les recomendó a los sujetos responder rápidamente y completar las oraciones con el contenido que prefirieran, pero asegurándose de producir una oración completa y aceptable. Además, se les solicitó que lo hicieran en el orden en el que se les presentaban y que no volvieran hacia atrás o releyeran lo escrito.

${ }^{4}$ Las dos condiciones difieren en varios sentidos que deben ser tenidos en cuenta en la comparación de los resultados entre ambas. Entre ellos, el grado de especificación de la referencia del sujeto de la subordinada (cf. n. 9) y la posible asociación de la secuencia pregunta-respuesta con una instancia de diálogo (respecto de la tendencia a la consistencia sintáctica o alineación en el diálogo, puede verse el trabajo de Branigan et al., 2000). 


\subsection{Grupo control:}

De manera complementaria, se evaluó la distribución de las preferencias de los sujetos por las diferentes estructuras en condiciones sin facilitación (grupo control). Para ello se pidió a 50 sujetos que completaran las mismas oraciones experimentales, esta vez sin las preguntas inductoras. Para evitar la facilitación entre respuestas, entre cada fragmento relevante se intercalaron cuatro fragmentos de relleno con diversas estructuras sintácticas no relacionadas, que también debían ser completados.

\section{RESULTADOS}

\subsection{Grupo control:}

En primer lugar se realizó la prueba control con las mismas oraciones. Las respuestas de los participantes fueron clasificadas de acuerdo a sus características categoriales en Sintagma de Flexión (SF), Sintagma de Complementante (SC), Sintagma Nominal (SN) y Otras. A partir de este análisis se estableció la frecuencia promedio de cada estructura respecto de los verbos críticos para los estímulos seleccionados. Se observó, así, que cada verbo daba lugar a una distribución idiosincrática de las estructuras seleccionadas. Esta distribución se utilizó como línea de base para la comparación con los resultados experimentales.

La Tabla I muestra la distribución de las preferencias de los sujetos control, es decir en condiciones sin facilitación. Así, por ejemplo, mientras los sujetos control completaron las oraciones que contenían el verbo prohibir con un SF en el $88 \%$ de los casos, sólo lo hicieron con un SF en el $23 \%$ de los casos cuando completaban oraciones con el verbo exigir. Asimismo, los SN nunca fueron los más frecuentes entre las opciones.

Tabla I. Porcentajes de las preferencias por SN, SF y SC en la prueba control.

\begin{tabular}{|l|l|l|l|}
\hline \multirow{2}{*}{ Verbo } & \multicolumn{3}{|l|}{ Porcentaje de respuestas $(\mathrm{N}=100)$} \\
\cline { 2 - 4 } & SF & SC & SN \\
\hline Propuso & 41 & 42 & 17 \\
\hline Recomendó & 57 & 33 & 10 \\
\hline Exigió & 23 & 51 & 26 \\
\hline Prohibió & 88 & 10 & 2 \\
\hline Permitió & 78 & 20 & 2 \\
\hline
\end{tabular}




\subsection{Resultados experimentales:}

\subsubsection{Condición I. (De comprensión a producción):}

Se analizaron en primer lugar los resultados de la Condición I, en la que el completamiento responde a una pregunta que los participantes leen previamente y se compararon las diferencias entre el rendimiento con prime SF y sin el prime (prueba control). La influencia del prime se midió utilizando una prueba de $\chi^{2}$ (con la corrección de Yates). En la Tabla II se muestran los resultados verbo por verbo. Si se considera el total de los verbos, se encontraron diferencias altamente significativas $\left(\chi^{2}=9.69 ; \mathrm{p}<0.01\right)$ entre los resultados de la condición control y la condición experimental. También se encontraron diferencias significativas al tomar en cuenta cada verbo independientemente, salvo en el caso del verbo prohibir $\left(\chi^{2}=0.02\right.$; $\mathrm{p}=0.92)$ y el verbo permitir $\left(\chi^{2}=1.12 ; \mathrm{p}=0.20\right)$. En estos casos puede verse que la frecuencia de completamiento de oraciones de la tarea control era cercana al límite superior ( $88 \%$ en el primer caso y $78 \%$ en el segundo), lo cual explicaría la poca o nula diferencia entre las condiciones con y sin prime (efecto techo).

Tabla II. Resultados del Experimento 1, Condición I con Prime SF comparados con grupo control.

\begin{tabular}{|l|l|l|l|}
\hline \multirow{2}{*}{ Verbo } & \multicolumn{2}{|l|}{$\%$ respuestas SF } & \multicolumn{1}{|l}{} \\
\cline { 2 - 4 } & Sin Prime & $\begin{array}{l}\text { Con Prime SF } \\
(\mathrm{N}=64)\end{array}$ & $\mathrm{p}$ \\
\hline Propuso & 41 & 67 & $0.00^{* *}$ \\
\hline Recomendó & 57 & 72 & $0.05^{*}$ \\
\hline Exigió & 23 & 48 & $0.00^{* *}$ \\
\hline Prohibió & 88 & 88 & 0.92 \\
\hline Permitió & 78 & 86 & 0.20 \\
\hline
\end{tabular}

En la misma Condición I, pero con el prime SC, se encontraron diferencias altamente significativas $(\mathrm{p}<0.01)$ entre los resultados de la tarea control y de la tarea experimental, en todos los casos, tal y como puede observarse en la Tabla III. 
Tabla III. Resultados del Experimento 1, Condición I con Prime SC comparados con grupo control.

\begin{tabular}{|l|l|l|l|}
\hline \multirow{2}{*}{ Verbo } & \% respuestas SC & \multirow{2}{*}{} \\
\cline { 2 - 4 } & Sin Prime & $\begin{array}{l}\text { Con Prime SC } \\
(\mathrm{N}=64)\end{array}$ & $\mathrm{p}$ \\
\hline Propuso & 42 & 70 & $0.00^{* *}$ \\
\hline Recomendó & 33 & 67 & $0.00^{* *}$ \\
\hline Exigió & 51 & 75 & $0.00^{* *}$ \\
\hline Prohibió & 10 & 61 & $0.00^{* *}$ \\
\hline Permitió & 20 & 48 & $0.00^{* *}$ \\
\hline
\end{tabular}

\subsubsection{Condición II. (De producción a producción):}

Se realizaron a continuación las mismas comparaciones para la Condición II. En este caso se observa la facilitación entre una oración previamente producida -el completamiento de la Condición I- respecto de un fragmento no relacionado que los participantes completan subsiguientemente. Para la Condición II con prime SF se encontraron diferencias significativas $(\mathrm{p}<0.05)$ con respecto a la prueba control para todos los verbos y el total, con excepción de los verbos proponer $\left(\chi^{2}=2.12\right.$; $\mathrm{p}=0.09)$, que presentó una significación que podría considerarse marginal, y prohibir $\left(\chi^{2}=0.01 ; \mathrm{p}=0.71\right)$. Los resultados se presentan en la Tabla IV.

Tabla IV. Resultados del Experimento 1, Condición II con Prime SF comparados con grupo control.

\begin{tabular}{|l|l|l|l|}
\hline \multirow{2}{*}{ Verbo } & \multicolumn{2}{|l|}{$\%$ respuestas SF } & \multicolumn{1}{|l}{} \\
\cline { 2 - 3 } & Sin Prime & Con Prime SF & p \\
\hline Propuso & 41 & $58(\mathrm{~N}=33)$ & $0.09^{(*)}$ \\
\hline Recomendó & 57 & $76(\mathrm{~N}=34)$ & $0.04^{*}$ \\
\hline Exigió & 23 & $43(\mathrm{~N}=23)$ & $0.04^{*}$ \\
\hline Prohibió & 88 & $86(\mathrm{~N}=42)$ & 0.71 \\
\hline Permitió & 78 & $93(\mathrm{~N}=42)$ & $0.03^{*}$ \\
\hline
\end{tabular}

En la Tabla V se muestran los resultados para la Condición II con prime SC y la condición control. Con excepción del verbo proponer $\left(\chi^{2}=1.09 ; \mathrm{p}=0.21\right)$, se observan diferencias altamente significativas entre las proporciones observadas en la condición experimental con prime SC y las observadas sin prime. 
Tabla V. Resultados del Experimento 1, Condición II con Prime SC comparados con grupo control.

\begin{tabular}{|l|l|l|l|}
\hline \multirow{2}{*}{ Verbo } & \multicolumn{2}{|l|}{ \% respuestas SC } & \multirow{2}{*}{} \\
\cline { 2 - 3 } & Sin Prime & Con Prime SC & p \\
\hline Propuso & 42 & $55(\mathrm{~N}=29)$ & 0.21 \\
\hline Recomendó & 33 & $59(\mathrm{~N}=29)$ & $0.01^{* *}$ \\
\hline Exigió & 51 & $78(\mathrm{~N}=37)$ & $0.00^{* *}$ \\
\hline Prohibió & 10 & $55(\mathrm{~N}=20)$ & $0.00^{* *}$ \\
\hline Permitió & 20 & $50(\mathrm{~N}=22)$ & $0.00^{* *}$ \\
\hline
\end{tabular}

3.2.3. Comparación entre resultados de Condición I y Condición II. Con el objetivo de estudiar las influencias de la tarea y el tipo de facilitador sobre el priming, se contrastó la magnitud del priming cuando éste resultaba de la lectura de la pregunta respecto de cuando resultaba del completamiento previo. Así, se comparó la diferencia de proporciones entre la Condición I y la Condición II, sin hallarse diferencias significativas $(\mathrm{p}>0.05)$ para ningún verbo ni para los totales.

\section{ANÁLISIS CUALITATIVO}

Por último, un análisis cualitativo detallado de las oraciones producidas en ambos experimentos indica que éstas difieren en contenido semántico de los primes, así como de otras respuestas anteriores. En otras palabras, se verificó que los blancos no están relacionados semánticamente más allá de las similitudes impuestas por la utilización del mismo verbo (incluyendo, claro, sus propiedades argumentales). Finalmente, el análisis indica que, en ambas condiciones, las estructuras internas de los completamientos también difieren de las de los inductores. Esto significa que la estructura detallada de los blancos no se vio afectada por la de los primes. De este modo, las oraciones completadas incluyeron segundos verbos con diferentes números de argumentos, así como toda clase de complementos y adjuntos, cuya presencia o ausencia no tuvo relación con las características de las oraciones facilitadoras 5 .

${ }^{5}$ Cabe notar también que, aun cuando en los primes con SC se presentó siempre la forma de subjuntivo en -e/ara (“¿qué les propuso que hicieran?”), en las respuestas se registró en ocasiones la variante en -e/ase ("les propuso que comprasen más mercadería”). 


\section{DISCUSIÓN}

De acuerdo con la definición del priming sintáctico, la presentación de una oración con una determinada configuración sintáctica modifica las preferencias de producción de los hablantes, de modo tal que se incrementa su tendencia a utilizar una oración con el mismo tipo de configuración a continuación. Según las dos perspectivas que buscan explicar este fenómeno, esta tendencia podría deberse a la activación residual de ciertas propiedades de los verbos -que fue el factor manipulado en nuestro experimento-, o bien a la repetición del patrón de asociación entre un mensaje y una estructura de constituyentes. Para verificar que este fenómeno tiene lugar en la producción de oraciones en español, diseñamos un experimento en el que después de leer o producir una oración cuya configuración sintáctica manipulamos sobre la base de las propiedades seleccionales de un verbo crítico, los participantes debían completar un fragmento no relacionado semánticamente, aunque con el mismo verbo.

En efecto, nuestros resultados muestran que es posible modificar las preferencias de los hablantes en relación con las alternativas seleccionales de un conjunto de verbos. Así, cuando experimentaban previamente un facilitador que incluía el verbo más un SF, los hablantes tendieron a completar la siguiente oración con esta construcción; y, complementariamente, lo hicieron en mayor proporción con un SC después de procesar oraciones con esta estructura.

Estos resultados no parecen explicables en términos de factores no sintácticos. En la medida en que facilitadores y blancos fueron presentados como parte de una serie de oraciones no relacionadas, una explicación de tipo discursivo no da cuenta de los datos. Tampoco una explicación basada en la semántica parece suficiente, en tanto las formas SF y SC son, al menos referencialmente, equivalentes. Tampoco una explicación léxica puede dar cuenta exhaustivamente de los resultados. La única fuente posible de superposición léxica es la repetición de la forma "que" en las respuestas SC. Sin embargo, un efecto de priming similar se obtiene en las respuestas $\mathrm{SF}$, donde no se da tal repetición.

Por otra parte, nos propusimos también comparar la magnitud del priming en dos condiciones: cuando facilitador y blanco son producidos por el participante (Condición II) y cuando el sujeto simplemente lee el prime, pero no tiene que producirlo (Condición I). Esta comparación nos permitió evaluar si el tipo de procesamiento del prime afectaba la magnitud del priming. La comparación no dio lugar a diferencias significativas ${ }^{6}$. En concordancia con hallazgos previos (Brani-

${ }^{6}$ Algunas otras características de los inductores y del tipo de procesamiento podrían estar modulando la respuesta a la facilitación. Entre ellos, la respuesta a la pregunta debe contribuir a la consistencia sintáctica local, asumiendo para la subordinada el sujeto que la pregunta propone. Se intentó controlar este efecto (que podría aumentar la tendencia a producir el mismo tipo de estructura) en 
gan, Pickering y Cleland, 2000; Potter y Lombardi, 1998; Weiner y Labov, 1983; Bock, 2002 y Bock, et al., 2007), este estudio mostró que el priming no sólo se produce tanto de producción a producción como de comprensión a producción, sino que la magnitud de la facilitación es indiferente del tipo de procesamiento del prime.

En relación con ello debe tenerse en cuenta que la situación experimental propuesta en la Condición I (en la que los sujetos no deben producir el prime, sino solamente leerlo) es la de pregunta-respuesta. En la medida en que la secuencia pregunta-respuesta reproduce las características típicas de la situación de diálogo, la consistencia de los resultados podría interpretarse en consonancia con los enfoques que enfatizan el papel del priming en la economía de recursos cognitivos.

La ocurrencia del efecto de comprensión a producción que se pone en evidencia en nuestros resultados $-y$ que se da también a la inversa (de producción a comprensión) $-^{7}$ sugiere que el locus del priming no es un proceso específico de una u otra modalidad, sino más bien un fenómeno debido a información que es compartida por ambas (Branigan et al., 1995; Branigan, 2007). Algunos autores han interpretado estos datos como soporte a la hipótesis de que el priming obedece a factores representacionales más que de procedimiento (los procedimientos involucrados en comprender una oración son diferentes de aquellos que intervienen en producirla; en cambio, habría elementos representacionales comunes). En esta línea, Branigan et al. (1995) (y trabajos posteriores hasta la actualidad) sostuvieron que el priming sintáctico surge como resultado de la activación residual de aspectos de esta representación. En este sentido, se sostiene, el priming es informativo respecto de la naturaleza de las representaciones léxicas.

El experimento manipuló las propiedades categoriales de los complementos de los verbos en las oraciones prime y mostró sensibilidad del efecto a la distinción SF/SC: aun cuando ambos tipos de sintagma codifican eventos, SC no prima SF, ni al revés, mientras que uno y otro se facilitan a sí mismos. Al mismo tiempo, las respuestas obtenidas en las pruebas de facilitación no se vieron afectadas por las características internas de los SF o SC. En otras palabras, el priming no fue sensible a la estructura detallada de estos constituyentes, mientras que sí lo fue a los rasgos categoriales de los núcleos, en concordancia también con los hallazgos de estudios anteriores. Tal como quedó establecido, el priming sintáctico no se basa sobre la repetición exacta de estructuras. Como en la investigación de Pickering

los estímulos, buscando que éstos admitieran siempre al menos dos posibilidades, como mínimo una de ellas compatible con la forma no primada.

${ }^{7}$ Utilizando una tarea de apareamiento oración-dibujo con oraciones con adjunción ambigua, Branigan et al. (2005) obtuvieron resultados comparables a los que se alcanzaron en contextos de producción. Los participantes de su estudio mostraron una mayor tendencia a producir un análisis en particular después de producir una descripción con la misma estructura. 
y Branigan (1998), expresiones que involucran la misma secuencia categorial de núcleo y complementos se facilitan unas a otras, incluso cuando la estructura interna de esos complementos difiere; por ejemplo cuando el SN post-verbal en las estructuras completivas difiere en términos de si contiene adjetivos, cláusulas relativas, etc.

Aunque el presente estudio no tuvo como objetivo comprobar el rol mediador de los elementos léxicos en el priming sintáctico, algunos de sus resultados pueden ser interpretados en este marco. De acuerdo con este enfoque, nuestros resultados sugieren, en conjunto, que la información codificada en los nodos combinatorios es de nivel sintagmático. Además, en tanto esta información es relevante para el priming, se infiere que los nodos combinatorios expresan la naturaleza categorial de los elementos que el verbo selecciona, es decir, si el sintagma seleccionado es de flexión o de complementante.

Sin embargo, los resultados muestran también que las estructuras más frecuentes no modificaron significativamente su participación en las respuestas. Este es el caso de los verbos prohibir y permitir, para los cuales la respuesta SF no mostró un aumento significativo cuando fue primada. Estos ejemplos puntuales podrían ser interpretados como un efecto del hecho de que exhiben valores extremos para SF en la prueba control (efecto techo). No obstante, es posible interpretar estos datos, consistentes con los otros resultados obtenidos, en el marco de una explicación más general que atiende al "estilo de procesamiento".

En este sentido, puede notarse también que la forma SC, la menos preferida para la mayoría de los verbos en la prueba control, obtuvo, en cambio, los mayores valores de facilitación. Se deduce de todo ello que las formas menos frecuentes son más sensibles al fenómeno del priming. En concordancia con ello, el verbo proponer, que distribuye sus preferencias más homogéneamente $(\mathrm{SF}=41 \%, \mathrm{SC}=42 \%$, $\mathrm{SN}=17 \%$ ) que los restantes casos considerados, obtuvo valores menos intensos de facilitación. Estos resultados reciben una interpretación bastante natural en el marco de un modelo del priming como aprendizaje implícito. De acuerdo con este enfoque, el priming consiste en el refuerzo de un patrón de asociación o bien en la elevación del nivel de reposo de una unidad en el sistema, con la contrapartida de la mengua en el nivel de reposo del resto de las unidades (Schade y Barattelli, 2001). El modelo, por lo tanto, predice que los patrones de asociación menos frecuentes serán más sensibles a los ajustes producidos en el sistema cada vez que son utilizados. En la medida en que la distribución es más homogénea, los ajustes producidos por la utilización de una unidad tendrán consecuencias menos notorias. Mayor investigación se requiere, sin embargo, en este campo para interpretar la cuestión como resultado de la competición o de la diferente sensibilidad de los valores de reposo.

Por último, este tipo de modelo predice también que el efecto es acumulativo. En este sentido, se esperaría que los participantes de nuestro experimento hubieran 
mostrado una mayor probabilidad de utilizar una estructura determinada si ésta había sido primada antes. Esta predicción, sin embargo, no se verifica en nuestros resultados. De acuerdo con ellos, los participantes no mostraron mayor probabilidad de utilizar una estructura si ésta había sido primada en el completamiento previo, ni la persistencia pareció extenderse más allá del completamiento inmediato posterior. En relación con los modelos del fenómeno de priming sintáctico, por lo tanto, nuestros resultados no discriminan entre las explicaciones alternativas. Más bien, aportan evidencia que recibe justificaciones parciales en ambos modelos. Sin embargo, como concluyen Ferreira y Bock (2006) y Branigan (2007), es plausible que el efecto tenga múltiples componentes cognitivos y que un modelo más comprehensivo, aún por desarrollar, deba contemplar la integración del papel de la activación de información léxica así como el de los mecanismos de aprendizaje implícito.

\section{CONCLUSIONES}

Hemos presentado un experiemento que, mediante una tarea de completamiento escrito de oraciones, muestra que el priming sintáctico se verifica en la producción de oraciones en español. Aun cuando diversos factores puedan superponerse para determinar la modificación de las tendencias de los participantes, parece concluyente que los estructurales tienen un peso preponderante. No obstante, factores que esperan ser estudiados en profundidad intervienen en la magnitud del efecto, como se sigue del hecho de que las estructuras marcadas - esto es las menos preferidas en condiciones sin facilitación- dan lugar a un priming más intenso. Por otra parte, como lo habían mostrado investigaciones previas, queda establecido en nuestro estudio que el priming se produce con la misma intensidad de comprensión a producción que cuando los participantes producen por sí mismos las oraciones facilitadoras.

Por último, en relación con la información sintáctica almacenada en las entradas léxicas de los verbos, hemos observado que las representaciones que codifican los nodos combinatorios son de nivel sintagmático y expresan la naturaleza categorial del complemento que el verbo selecciona.

\section{REFERENCIAS}

Bock, K. 1986. "Syntactic persistence in language production". En Cognitive Psychology, 18, 355-387.

Bock, K. 1989. "Closed-class immanence in sentence production". En Cognition, 31, 163-186. 
Bock, K. 2002. "Persistent structural priming from comprehension to production". Presentado en CUNY Conference on Human Sentence Processing, Nueva York, marzo de 2002.

Bock, K., Dell, G. S., Chang, F. y Onishi, K. 2007. "Structural persistence from language comprehension to language production". En Cognition, 104 (3), 437-458.

Bock, J. K. y Griffin, Z. M. 2000. "The persistence of structural priming: Transient activation or implicit learning?” En Journal of Experimental Psychology: General, 129, 177-192.

Bock, K. y Loebell, H. 1990. "Framing sentences”. En Cognition, 35, 1-39.

Bock, J. K., Loebell, H. y Morey, R. 1992. "From conceptual roles to structural relations: Bridging the syntactic cleft". En Psychological Review, 99, 150-171.

Branigan, H. 2007. "Syntactic Priming”. En Language and Linguistics Compass, 1 (12), 1-16.

Branigan, H., Pickering, M. y Cleland, A. 2000. "Syntactic co-ordination in dialogue". En Cognition, 75, B13-25.

Branigan, H.P., Pickering, M. J., Liversedge, S., Stewart, A. y Urbach, T. 1995. "Syntactic priming: Investigating the mental representation of language". En Journal of Psycholinguistic Research, 24 (6), 489-506.

Branigan, H.P., M.J. Pickering y McLean, J.F. 2005. "Priming prepositional-phrase attachment during comprehension". En Journal of Experimental Psychology: Learning, Memory and Cognition, 31, 468-481.

Chang, F., Dell, G. S., Bock, K. y Griffin, Z. M. 2000. "Structural priming as implicit learning: A comparison of models of sentence production”. En Journal of Psycholinguistic Research, 29 (2), 217-229.

Corley, M. M. B. y Scheepers, C. 2002. "Syntactic priming in English sentence production: Categorical and latency evidence from an internet-based study". En Psychonomic Bulletin \& Review, 9 (1) 126-131.

Ferreira, V. 2003. "The persistence of optional complementizer production: Why saying 'that' is not saying 'that' at all'. En Journal of Memory and Language, 48 (2), 379-398.

Ferreira V. y Bock, K. 2006. “The functions of structural priming”. En Language and Cognitive Processes, 21 (7-8), 1011-1029.

Hartsuiker, R.J. y Kolk, H.H.J. 1998. "Syntactic persistence in Dutch". En Language and Speech, 41 (2), 143-184.

Hartsuiker, R.J., Kolk, H.H.J. y Huiskamp, P. 1999. "Priming word order in sentence production”. En Quarterly Journal of Experimental Psychology A: Human Experimental Psychology, 52A (1), 129-147.

Hartsuiker, R.J. y Westenberg, C. 2000. "Word order priming in written and spoken sentence production." En Cognition, 75 (2), 27-39.

Herranz, M.L. 1999. “El infinitivo”. En Bosque, I. y Demonte, V. (comps.) Gramática descriptiva de la lengua española. Madrid: Espasa.

Kempen, G. 1977. "Conceptualizing and formulating in sentence production". En: Rosenbaum, S. (ed.) Sentence production: Developments in research and theory. 
Hillsdale, NJ: Lawrence Erlbaum Associates Inc.

Levelt, W.J.M., Roelofs, A. y Meyer, A.S. 1999. "A theory of lexical access in speech production". En Behavioral and Brain Sciences, 22, 1-75.

Melinger, A. y Dobel, C. 2005. "Lexically-driven syntactic priming". En Cognition, 98, B11-B20.

Pickering, M. y Branigan, H. 1998. "The representation of verbs: Evidence from syntactic priming in language production". En Journal of Memory and Language, 39, 633-651.

Pickering, M. J. y Branigan, H. P. 1999. "Syntactic priming in language production". En Trends in Cognitive Sciences, 3 (4), 136-141.

Pickering, M. J. y Garrod, S. 2004. "Towards a mechanistic Psychology of dialogue". En Behavioral and Brain Sciences, 27, 169-226.

Potter, M. C. y Lombardi, L. 1998. "Syntactic priming in immediate recall of sentences". En Journal of Memory and Language, 38 (3), 265-282.

Roelofs, A. 1992. "A spreading-activation theory of lemma retrieval in speaking". En Cognition, 42, 107-142.

Roelofs, A. 1993. "Testing a non-decompositional theory of lemma retrieval in speaking: Retrieval of verbs". En Cognition, 47, 59-87.

Schade, U. y Barattelli, S. 2001. "Never Change a Winning Team: Towards Modelling Syntactic Priming in Language Production". [En línea]. Disponible en http:// www.lili.uni-bielefeld.de/ -schade/cogsci01.ps [Consulta: mayo de 2007; texto completo accesible en http://citeseer.ist.psu.edu/478339.html.]

Scheepers, C. 2003. "Syntactic priming of relative clause attachments: Persistence of structural configuration in sentence production”. En Cognition, 89, 179-205.

Schenkein, J. 1980. "A taxonomy for repeating action sequences in natural conversation”. En: B. Butterworth (comp.), Language production, Vol. 1, 21-47. Londres: Academic Press.

Sevilla, Y., Jaichenco, V., Olmedo, A. y Raiter, A. 2006. "El papel de la información léxica en el priming sintáctico. Una experiencia con verbos aislados”. En: Memorias de las XIII Jornadas de Investigación. Segundo encuentro de investigadores en Psicología del MERCOSUR. Tomo III, 474-476. Buenos Aires: Universidad de Buenos Aires.

Smith, M. y Wheeldon, L. 2001. "Syntactic priming in spoken sentence production: An online study". En Cognition, 78, 123-164.

Tannen, D. 1989. Talking voices: Repetition, dialogue and imagery in conversational discourse. Cambridge: Cambridge University Press.

Trueswell, J. C. y Kim, A. E. 1998. "How to prune a garden-path by nipping it in the bud: Fast-priming of verb argument structures". En Journal of Memory and Language, 39, 102-123.

Weiner, J. y Labov, W. 1983. "Constraints on the agentless passive". En Journal of Linguistics 19, 29-58. 